\title{
DOPPLER FETOPLACENTARIO EN HIPERTENSIÓN GESTACIONAL Y PREECLAMPSIA LEVE
}

Alexandra Casasbuenas MD*, José Luis Rojas MD**, Juan Carlos Bello-Muñoz MD***

\section{Resumen}

Introducción: la preeclampsia se presenta en $6 \%$ de los embarazos en Colombia y se asocia con una elevada tasa de morbimortalidad maternofetal. Con frecuencia los casos severos se acompañan de restricción del crecimiento intrauterino; en estos casos el doppler fetoplacentario es útil para determinar el pronóstico fetal, pero su valor en preeclampsia leve o hipertensión gestacional no está bien definido. Objetivos: determinar en estas dos circunstancias la frecuencia de alteraciones en el doppler de las arterias umbilical y cerebral media. Materiales y métodos: búsqueda de casos en la base datos de la unidad de medicina maternofetal del Hospital de San José, entre agosto de 2006 y febrero de 2008. Se definió como casos las pacientes con gestaciones $\geq$ a 28 semanas con HTG o PL y fetos con perfil de crecimiento normal. Se consideraron y analizaron variables demográficas, resultados del doppler fetoplacentario y las complicaciones. Resultados: se identificaron 85 pacientes. El $17 \%$ presentó alteración del doppler de la AU y 7\% de la ACM; en gestaciones < 32 semanas los hallazgos anormales son bajos (75\% AU y 85\% ACM normales). Conclusiones: la frecuencia de alteraciones en el Doppler de AU y ACM fue similar en el grupo de mujeres que presentaron complicaciones y aquellas con un desenlace normal.

Palabras clave: preeclampsia, embarazo, hipertensión, bajo peso al nacer, apgar, doppler de la arteria umbilical, doppler de la arteria cerebral media.

Abreviaturas: RCIU, restricción del crecimiento intrauterino; PL, preclampsia leve; HTG, hipertensión gestacional; AU, arteria umbilical; ACM, arteria cerebral media.

\section{THE USEFULNESS OF FETAL PLACENTAL DOPPLER ULTRASOUND IN GESTATIONAL HYPERTENSION AND MILD PREECLAMPSIA}

\section{Abstract}

Introduction: In Colombia, around 6\% of pregnant women will experience preeclampsia. It is associated with high maternal and fetal morbidity and mortality rates. Severe cases are frequently related to intrauterine growth restriction; placental and fetal Doppler has prognostic value in these cases, although, its usefulness in mild preeclampsia (MP) or gestational hypertension (GHT) is not yet well defined. Objectives: to determine the frequency of abnormal Doppler sonography of the umbilical artery (UA) and middle cerebral artery (MCA) in MP or GHT. Materials and methods: cases were searched for in the Maternal Fetal Unit databases at Hospital de San José between August 2006 and February 2008. Cases were defined as patients with $\geq 28$ week pregnancies complicated by GHT or MP with fetuses with a normal growth profile. Demographic variables, placental fetal Doppler evaluation results and complications were considered and analyzed. Results: 85 patients were identified. Seventeen percent (17\%) presented abnormal Doppler of the UA and 7\% of the MCA; abnormal findings were low (normal UA in $75 \%$ and normal MCA in $85 \%$ ) in $<32$ week gestations. Conclusions: the frequency of abnormal Doppler results of the UA and MCA were similar in the group of women with complications and in those who had a normal pregnancy outcome.

Key words: preeclampsia, pregnancy, hypertension, low birth weight, Apgar scores, umbilical artery Doppler, middle cerebral artery Doppler.

Fecha recibido: noviembre 12 de 2009-Fecha aceptado: febrero 18 de 2010

* Residente de Medicina Maternofetal. Fundación Universitaria de Ciencias de la Salud. Hospital de San José, Bogotá DC. Colombia.
** Especialista en Medicina Maternofetal. Instructor Asociado, Fundación Universitaria de Ciencias de la Salud. Bogotá DC. Colombia.

*** Unidad medicina fetal. Departamento de Ginecología y Obstetricia, Hospital Universitari Vall d’Hebron, Universidad Autónoma de Barcelona, España. 


\section{Introducción}

La preeclampsia es una patología que incrementa el riesgo de complicaciones maternas y fetales. ${ }^{1}$ La prevalencia de esta enfermedad alcanza el $2 \%$ en los países industrializados, mientras que en aquellos en vía de desarrollo se presenta hasta en el $7 \%$ de la población gestante. ${ }^{2,3}$ De manera global se ha descrito como la causa de muerte en $15 \%$ a $20 \%$ de las embarazadas, cifra que alcanza el $42 \%$ en nuestro país. ${ }^{1}$ La etiología de esta entidad continúa desconocida después de años de investigación; en la actualidad se acepta el papel preponderante de la placentación inadecuada, que parece estar mediada por una segunda etapa incompleta de migración trofoblástica que genera una serie de alteraciones en la fisiología de la circulación del lecho placentario. ${ }^{1,2,4}$ Estos cambios promueven un daño endotelial sistémico que representa el punto de partida de la enfermedad y las complicaciones asociadas, entre las que se encuentra RCIU., ${ }^{1,4-12}$

La preeclampsia representa cerca del $40 \%$ de las causas de origen materno relacionadas con RCIU. ${ }^{4}$ En las mujeres que presentan estas dos patologías se ha encontrado que la placenta exhibe alteraciones en el desarrollo de las vellosidades terminales, que resultan ser más pequeñas y estar obliteradas e hiperplásicas; la pobre vascularización regional dificulta el intercambio gaseoso generando un desorden endotelial que produce variaciones en la circulación materna y fetal. ${ }^{4-7,10,12}$ Estos cambios disminuyen el aporte de oxígeno y de nutrientes al feto, lo cual se refleja en una disminución de la tasa de crecimiento, por lo que resulta coherente pensar que para que el perfil de desarrollo fetal se altere de manera significativa, la circulación placentaria también debe estar comprometida. ${ }^{3,4,6,13,14}$

La predicción de complicaciones asociadas con la preeclampsia ha sido un tema difícil de explorar. Se han propuesto numerosas pruebas serológicas e imagenológicas, pero la gran mayoría han resultado inútiles. El estudio de la circulación materna y fetal por medio del análisis espectral del doppler es el método más aceptado para lograr este objetivo en embarazos de alto riesgo. ${ }^{15}$ Por ello, como parte del manejo rutinario de las embarazadas con fetos que presentan RCIU, se ha pro- puesto la realización del doppler de la AU y ACM que permitan detectar de manera oportuna la presencia de insuficiencia placentaria con o sin adaptaciones hemodinámicas fetales. ${ }^{7}$ No obstante, el doppler de la AU y el perfil de crecimiento fetal no se modifican en presencia de un compromiso menor de la circulación placentaria, circunstancia que ocurre con mayor frecuencia en casos de HTG y PL. ${ }^{3,13,16-18}$

Es clara la asociación entre las alteraciones del flujo de la AU y el pobre resultado perinatal en un embarazo de alto riesgo. ${ }^{15}$ Estas variaciones reflejan cambios hemodinámicos que se asocian con la adaptación del feto al inadecuado funcionamiento de la placenta y algunos casos manifiestan RCIU secundario. La utilización del doppler en poblaciones con preeclampsia severa ha permitido disminuir la muerte perinatal (OR 0.71, IC95\% 0.7-0.93), pese a esto, el uso rutinario en embarazos de bajo riesgo no ha demostrado mayor utilidad. ${ }^{3,12,15}$ Es razonable considerar que la utilidad del doppler para determinar el pronóstico fetal en pacientes con PL leve o HTG que presentan un perfil de crecimiento fetal adecuado y aquellas que presentan un estado severo con o sin compromiso del crecimiento fetal es diferente. . $^{5,16-19}$ En el primero no es clara la utilidad de la prueba diagnóstica para predecir las complicaciones fetales y de manera específica las alteraciones en el perfil de crecimiento fetal. El objetivo del presente estudio fue determinar la frecuencia de alteraciones en el doppler de AU y ACM en una población con diagnóstico de PL o HTG y analizar los resultados de acuerdo con los desenlaces maternos y fetales.

\section{Materiales y métodos}

Se revisó la base de datos del servicio de ecografía de la unidad de medicina maternofetal del Hospital de San José, durante el período comprendido entre agosto 2006 y febrero 2008. Se identificaron las pacientes con estados hipertensivos del embarazo a quienes se les realizó doppler de AU y ACM. Después se tomaron en cuenta aquellas con HTG y PL que tuviesen fetos con perfil de crecimiento normal (definido como el estimado por encima del percentil 10) que presentaran edad gestacional $\geq 28$ semanas en el momento del diagnóstico. Las evaluacio- 
nes ultrasonográficas se realizaron utilizando un equipo Toshiba Exario. Se definió PL como la presencia de cifras tensionales mayores o iguales a 140/90 mm Hg en presencia de proteinuria aislada mayor o igual a $75 \mathrm{mg} /$ $\mathrm{dL}$ o en 24 horas superior a $300 \mathrm{mg} / \mathrm{dL}$, e HTG como la presencia de cifras tensionales mayores o iguales a 140/ $90 \mathrm{~mm} \mathrm{Hg}$ sin evidencia de proteinuria.

No se consideraron las pacientes con preeclampsia severa determinada por la presencia de síntomas clínicos de vasoespasmo, elevación de transaminasas, trombocitopenia (recuento de plaquetas inferior o igual a 150.000) y oligohidramnios (índice de líquido amniótico menor o igual a 6); tampoco aquellas con malformaciones fetales mayores (alteraciones en la formación de un órgano o tejido que comprometa la vida del feto), diabetes mellitus pregestacional y embarazo múltiple.

La información extraída de las historias clínicas fue valorada de manera independiente por dos investigadores, quienes registraron los datos en un formato diseñado para el estudio; después fueron digitados en una base de datos donde se evaluaron usando el paquete estadístico $S P S S^{\circledR} 12.0$ (licencia FUCS, SPSS Inc. Headquarters, Chicago, IL). Se consideraron y analizaron diversas variables demográficas como edad gestacional cuando se diagnosticó el estado hipertensivo del embarazo y la del parto, los índices de pulsatilidad de AU y ACM, que se consideraron anormales si el de la AU se encontraba por encima del P 95 para la edad gestacional y el de la ACM por debajo del P 5. Así mismo, se evaluaron las complicaciones del embarazo, el peso al nacer, el apgar al minuto y a los cinco minutos y la duración de la hospitalización del recién nacido en la unidad de recién nacidos, así como la mortalidad materna y perinatal. En los casos en los que continuó el manejo de la paciente en otra entidad o el parto se atendió en otra institución, se realizó contacto por vía telefónica para completar la información. No se consideró necesaria la obtención de consentimiento informado y el protocolo fue aprobado por el comité de investigaciones y ética de la Fundación Universitaria de Ciencias de la Salud. Los resultados fueron descritos por medio de la determinación de frecuencias absolutas, relativas, medidas de tendencia central y de dispersión.

\section{Resultados}

Se identificaron 122 pacientes con estados hipertensivos del embarazo a quienes se les practicó un doppler de la AU y ACM; de éstas, se seleccionaron 85 que cumplieron la definición de caso. Cuarenta y cuatro (51\%) presentaron PL al ingresar al estudio y las restantes 41 (49\%) HTG. La media de la edad gestacional al momento del diagnóstico del estado hipertensivo fue de 33 semanas (DE +/-3), siendo el límite inferior de 28 y el superior de 40. Cerca de la cuarta parte de la población $(n=20)$ ingresó al estudio con una edad gestacional menor de 32 semanas. El promedio de la edad gestacional al momento del parto fue de 35.7 semanas (DE +/-3.14) y en diez pacientes (12\%) el parto se presentó antes de la semana 32. El promedio del peso al nacer fue $2.360 \mathrm{~g}(\mathrm{DE}+/-$ $682 \mathrm{~g})$ y el de la talla $46 \mathrm{~cm}(\mathrm{DE}+/-4 \mathrm{~cm})$.

La muerte fetal en la semana 29 se produjo como consecuencia de un abrupcio de placenta, este feto y los fallecimientos neonatales ocurrieron en casos con edad gestacional menor de 32 semanas. Cinco de las seis pacientes que presentaron muerte de los recién nacidos progresaron a preeclampsia severa en los cinco días posteriores al ingreso hospitalario, una de ellas cursó con eclampsia y otra presentó síndrome de Hellp. El promedio del apgar al minuto fue $8(\mathrm{DE}+/-1.3)$ y a los cinco minutos 9 (DE +/- 1.3). Entre los recién nacidos hospitalizados dos demostraron alteraciones de ACM con índice de pulsatilidad de AU elevado, se diagnosticaron en la semana 28 y evolucionaron a preeclampsia severa en la semana siguiente al diagnóstico. En ambos casos la gestación finalizó durante la semana de hospitalización y los recién nacidos murieron en los primeros 28 días de vida.

No se encontró ninguna muerte materna; 21 presentaron complicaciones, 14 por progresión a preeclampsia severa, cinco por síndrome de Hellp y dos por eclampsia. En 13 mujeres (62\%) las complicaciones se diagnosticaron antes de la semana 32 de gestación. Del total de la población estudiada, en quince casos (17\%) hubo alteración del doppler de la AU; en dos de estas mujeres se encontró ausencia del flujo en diástole y en una inversión del mismo, y la ACM se encontró alterada en seis $(7 \%)$ de los casos, tres evolucionaron a preclampsia severa y una a síndrome de Hellp; ade- 
más, en el grupo con doppler de ACM y AU alterados no se presentaron muertes fetales y dos de ellas fueron hospitalizadas en la UCI. El de la ACM se encontró alterado en 4/44 (9\%) y en 2/41 (5\%) en PL e HTG respectivamente. En la Tabla 1 se presentan las características de las pacientes de acuerdo con la presencia de doppler AU normal o anormal.

Las Figuras 1 y 2 muestran las distribuciones del índice de pulsatilidad de AU y ACM de acuerdo con la edad gestacional. Para el subgrupo de mujeres con menos de 32 semanas de embarazo $(n=10)$ el doppler de AU fue normal en seis (60\%) y el de ACM en siete (70\%) (Tabla 1). Sólo una paciente, cuyo recién nacido requirió ingreso a la unidad de cuidados intensivos, se desembarazó por alteraciones en el doppler fetoplacentario, en el que se encontró aumento en los índices de resistencia y pulsatilidad de AU, vasodilatación cerebral y flujo pulsátil en la vena umbilical. Aunque la curva de crecimiento fetal fue normal, 23 casos (27\%) presentaron bajo peso al nacer; de éstos, 17 (74\%) tuvieron doppler de AU normal, y entre los 51 casos con adecuado peso al nacer, 43 (84\%) causaron con doppler de AU normal. La ACM no se encontró alterada en $21 / 23(91,3 \%)$ pacientes con bajo peso y en $45 / 49(91,8 \%)$ con adecuado peso al nacer (Tabla 1). A pesar del esfuerzo por obtener la información completa de las pacientes atendidas en otras instituciones el porcentaje de información perdida con relación a los resultados perinatales en el estudio fue de $8 \%$.

\section{Discusión}

La preeclampsia representa una de las patologías con mayor riesgo materno y fetal. El impacto del doppler fetoplacentario en la evaluación del pronóstico fetal en pacientes con formas leves de la enfermedad es difícil de determinar; por el momento, ha demostrado ser útil para disminuir la probabilidad de muerte fetal en preeclampsia severa. En gestaciones de bajo riesgo Briker y Neilson encontraron que el uso rutinario del doppler fetoplacentario no tiene repercusiones sobre el resultado

\begin{tabular}{|c|c|c|c|}
\hline $\begin{array}{l}\text { Variables } \\
\text { *Promedio (DE) }\end{array}$ & $\begin{array}{c}\text { Doppler AU anormal } \\
\text { n (\%) } \\
15(17,6)\end{array}$ & $\begin{array}{c}\text { Doppler AU normal } \\
\text { n (\%) } \\
70(82,3)\end{array}$ & $\begin{array}{l}\text { Total } \\
85\end{array}$ \\
\hline PL & $7(16)$ & $37(84)$ & $44(51)$ \\
\hline HTG & $8(20)$ & $33(80)$ & $41(49)$ \\
\hline EG en el momento del diagnóstico sem* & $33(3,8)$ & $34(2,7)$ & $33(3)$ \\
\hline Diagnóstico $\leq 32 \mathrm{sem}$ & $15(75)$ & $5(25)$ & $20(23)$ \\
\hline Parto $<37$ sem & II (23) & $37(77)$ & $48(56)$ \\
\hline Parto $<32$ sem & $4(40)$ & $6(60)$ & $10(12)$ \\
\hline $\begin{array}{l}\text { Peso al nacer g *(promedio DE) } \\
\text { (describir peso min y máximo) }\end{array}$ & $1878(689,3)$ & $2473(634,2)$ & $2360(682)$ \\
\hline Pequeño para edad gestacional & 6 & 17 & $23(27 \%)$ \\
\hline Talla $\mathrm{cm} *$ & $44(4,5)$ & $46(4,3)$ & $46(4)$ \\
\hline Requerimiento URN (SI) & $7(25)$ & $21(75)$ & $28(33)$ \\
\hline Total de complicación materna & $4(19)$ & $17(81)$ & $21(25)$ \\
\hline Muerte fetal (SI) & 0 & I (100) & $I(I, 2)$ \\
\hline Muerte neonatal (SI) & $3(50)$ & $3(50)$ & $6(7)$ \\
\hline
\end{tabular}

* PL, preeclampsia leve; HTG, hipertensión gestacional; EG, edad gestacional; DE, desviación estándar; sem, semanas; g, gramos; $\mathrm{cm}$, centímetros; URN, unidad de recién nacidos. 


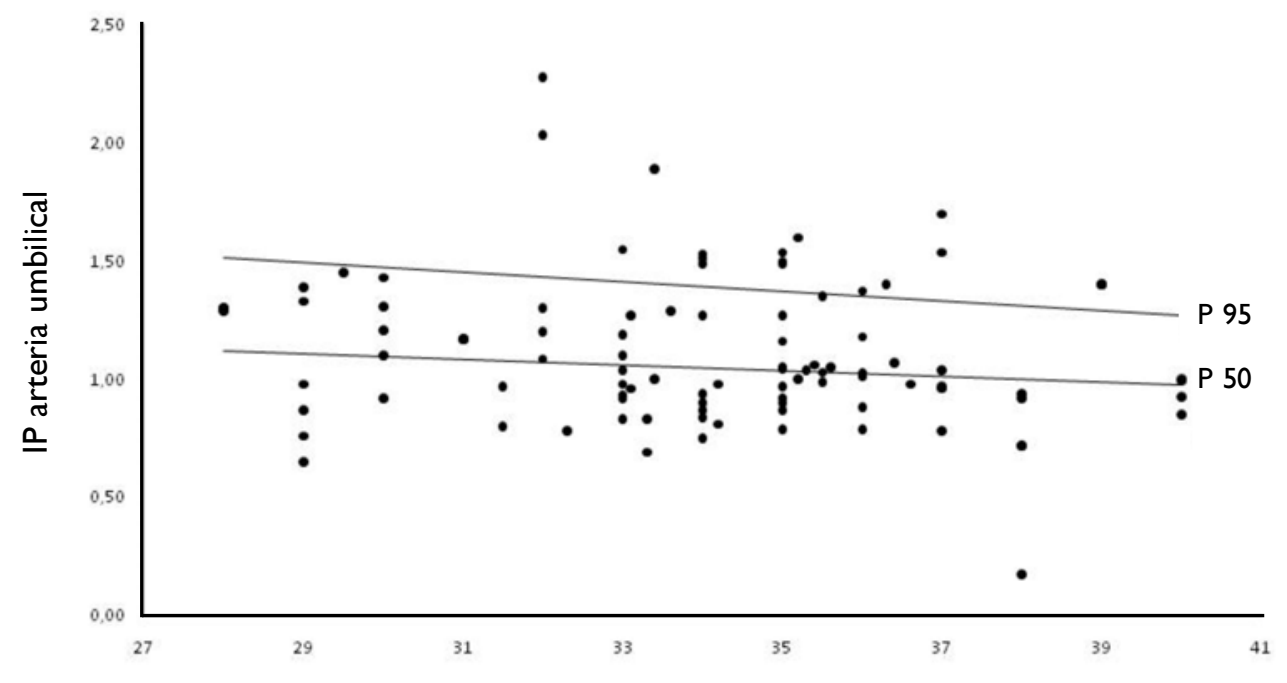

Edad gestacional

Figura I. Distribución del IP de la AU de acuerdo con la edad gestacional.

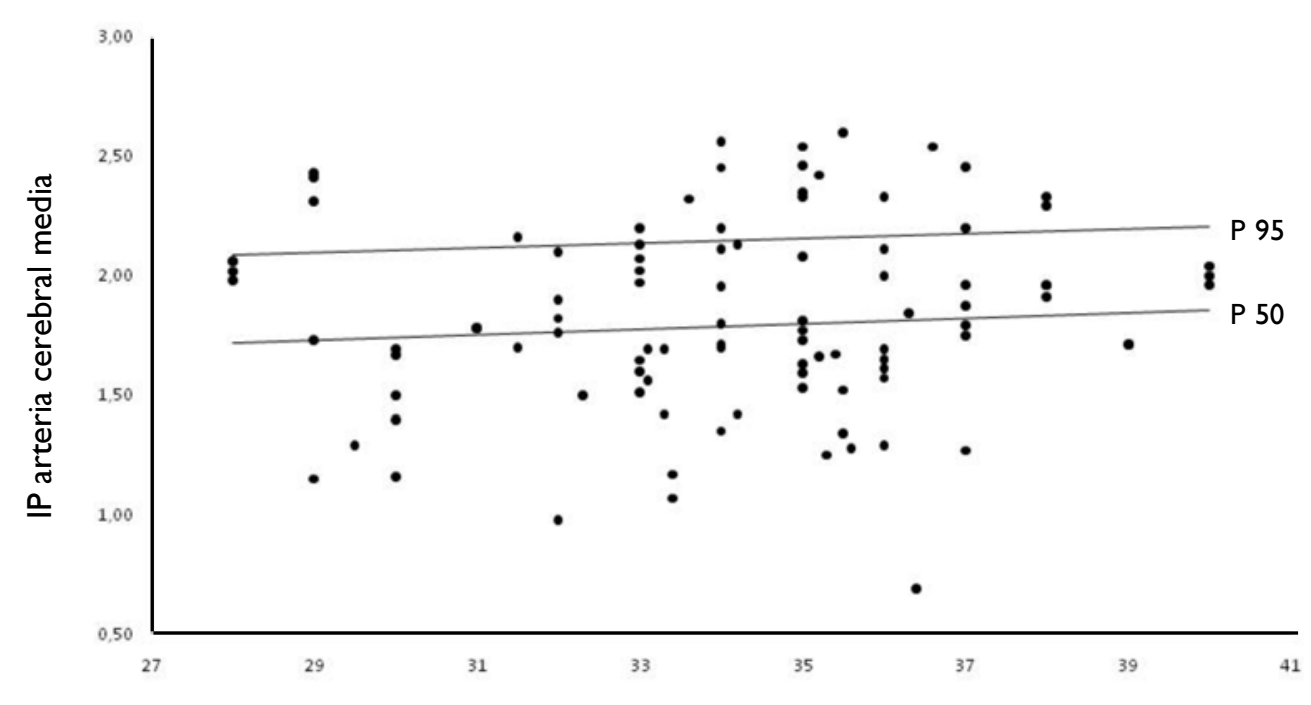

Edad gestacional

Figura 2. Distribución del IP de la ACM de acuerdo con la edad gestacional. 
materno y fetal. ${ }^{3,15,20}$ A pesar de ser una patología que se presenta hasta en el $6 \%$ de las gestaciones en Colombia, la preeclampsia severa asociada con parto antes de la semana 34 y aumento significativo de los riesgos inherentes a la prematurez, ocurre sólo en $0,5 \%$ de las mujeres. ${ }^{1,16}$ Las pacientes que presentan PL o HTG tienen una menor prevalencia de complicaciones maternas y fetales; sin embargo, su evaluación inicial es básica para el manejo y la toma de decisiones posteriores que determinan el pronóstico de la gestación.

En general, las lesiones placentarias en preeclampsia muestran una relación directa entre la arteriopatía decidual, los infartos e hipermaduración de las vellosidades y la edad gestacional en el momento del parto. ${ }^{15,17}$ Para algunos autores la PL que se presenta en un embarazo a término y la severa que se encuentra en edades gestacionales tempranas o a término asociada con RCIU, representan dos entidades diferentes. ${ }^{18-21} \mathrm{El}$ primer grupo de pacientes puede tener una asociación leve o ninguna, con el desarrollo deficiente del trofoblasto. Dicha hipótesis también está respaldada por la observación de que los productos de mujeres con PL o HTG por lo regular presentan pesos normales o grandes para su edad. ${ }^{21}$ Se ha sugerido que el síndrome que desencadena la preeclampsia corresponde desde el punto de vista fisiopatológico a una vía común en la cual terminan las dos entidades, una más severa asociada con la formación deficiente del trofoblasto que compromete el crecimiento fetal y la segunda, relacionada con cambios escleróticos en las arteriolas espirales. ${ }^{16,18}$ En esta serie de casos se observó que el doppler fetoplacentario no se alteró con mayor frecuencia en PL o HTG que después presentaron alguna complicación materna o fetal, entre ellas el bajo peso al nacer. La prevalencia de muerte neonatal fue del $7 \%$ en embarazos menores de 32 semanas, por lo que puede atribuirse en primera instancia a la prematurez de los recién nacidos; sin embargo, es interesante anotar que sólo el $25 \%$ presentó doppler de AU anormal y el $85 \%$ ACM sin alteraciones. Es razonable cuestionar el valor del doppler de AU y ACM en la predicción de los resultados perinatales de un PL o HTG con perfil de crecimiento fetal normal. Es interesante notar que las pacientes con edades gestacionales tempranas que presentan una forma leve de la enfermedad con doppler fetoplacentario normal, pueden evolucionar en los siguientes días a formas más severas, posiblemente como consecuencia de la naturaleza dinámica de los estados hipertensivos de la gestación. ${ }^{22}$ Casi todas las pacientes se desembarazaron por complicaciones maternas secundarias a su patología de base y sólo una por alteraciones en el doppler, lo que sugiere que los resultados de este análisis en este grupo no predice el desenlace materno ni fetal. Tampoco parece modificar el enfoque clínico, el manejo ni el resultado materno o fetal, pues esta prueba diagnóstica evalúa las características hemodinámicas del momento en que se realiza y es difícil establecer el grado de compromiso endotelial subyacente que puede evolucionar hacia mayor severidad. En nuestra opinión las gestaciones complicadas por cualquier estado hipertensivo que se presenten antes de la semana 32 deben ser controladas de manera estricta, sin basarse sólo en los hallazgos del doppler de la AU o de la $\mathrm{ACM}$, los cuales en caso de ser normales no son tranquilizadores.

El desarrollo de RCIU es un proceso crónico que depende del compromiso de la circulación placentaria en primera instancia y de manera secundaria del intercambio gaseoso y de nutrientes. En este estudio, el $27 \%$ de los recién nacidos presentaron bajo peso al nacer a pesar de que se seleccionaron mujeres con embarazos que se encontraban en el tercer trimestre con un perfil de crecimiento ecográfico por encima del percentil 10. Este hallazgo puede explicarse por la evaluación de las características de las pacientes en un momento determinado; por lo tanto, los datos con relación a la curva de crecimiento en el transcurso de la gestación es limitado, con un grupo de recién nacidos que presentó disminución posterior de su tasa de crecimiento.

Tampoco se encontraron diferencias en el doppler de aquellos fetos con crecimiento adecuado o bajo peso al nacer. Esto permite suponer que a pesar de los hallazgos de normalidad del doppler, el crecimiento de estos fetos debe ser vigilado. Los hallazgos del doppler fueron similares en relación con otras complicaciones neonatales severas, ya que el $33 \%$ de los neonatos requirieron traslado a la unidad de cuidados intensivos y sólo el $25 \%$ de éstos presentaron alteraciones del doppler de $\mathrm{AU}$ y el $7 \%$ en la ACM. Todas las muertes perinatales se presentaron en recién nacidos productos de partos a la semana 32 de embarazo, es muy posible que sea consecuencia de la prematurez de los fetos y no como resulta- 
do de las alteraciones asociadas con el compromiso vascular propio de la preeclampsia, lo que explica que no puedan ser anticipadas con el doppler fetoplacentario.

Los resultados de este estudio generan dudas en relación con la utilidad del doppler fetoplacentario en mujeres que se encuentran en el tercer trimestre de la gestación y que presentan PL o HTG con crecimiento fetal normal. Este hallazgo concuerda con los resultados reportados en la revisión sistemática realizada por Neilson y colaboradores con estudios que han tenido en cuenta poblaciones no seleccionadas de bajo riesgo. ${ }^{15}$ Así, no se encontró una mayor frecuencia de alteraciones en el doppler de pacientes con complicaciones al compararlas con aquellas sin complicaciones.

Es importante resaltar que el personal que controla de forma rutinaria a estas pacientes debe estar entrenado para evitar la realización indiscriminada de estudios que generen información confusa que pueda conducir a la toma de decisiones clínicas inadecuadas o apresuradas. Además los resultados de este estudio deben analizarse considerando su carácter retrospectivo, lo cual no permite el seguimiento de las características clínicas de las pacientes y del crecimiento de los fetos en los casos manejado en otras instituciones. Por otro lado, también se deben considerar las limitaciones secundarias a la pérdida de la información y el pequeño grupo de pacientes. Para obtener conclusiones definitivas con relación al valor pronóstico del doppler fetoplacentario en un grupo como éste, se requiere de un estudio prospectivo diseñado con este fin.

\section{Referencias}

1. López-Jaramillo P. Bioquímica del endotelio vascular: implicaciones fisiológicas y clínicas. 5a ed. Bogotá: Horizonte impresores; 2001

2. Norwitz ER. Implantation and the survival of early pregnancy. N Eng J Med 2001;59(6):1400-18

3. Spectral Doppler sonography, wave form analysis and hemodynamic interpretation. In: Maulik D, editor. Doppler ultrasound in obstetrics and gynecology. New York: Springer - Verlag; 1997. p. 55.

4. Pridjian G, Puschett JB. Clinical and pathophysiologic considerations of preeclampsia. Obs Gynecol Surv. 2002; 57(9):598-618.

5. Walker JJ. Preeclampsia. Lancet. $2000 ; 356(9237): 1260-5$

6. Pridjian G, Puschett JB. Preeclampsia part 2: experimental and genetic considerations. Obstet Gynecol Surv. 2002; 57(9): 619-40.
7. Wilson ML, Goodwin TM, Pan VL, Ingles SA. Molecular epidemiology of preeclampsia. Obst Gynecol Surv. 2002; 58(1):1158-68

8. Page NM. The endocrinology of pre-eclampsia. Clin Endocrinol (Oxf). 2002; 57(4):413-23.

9. Gillham JC. An overview of endothelium-derived hyperpolarising factor (EDHF) in normal and compromised pregnancies. Eur J Obst Gynecol Rep Biol. 2003;109:2-7

10. Arslan M. Endothelin-1 and leptin in the pathophysiology of intrauterine growth restriction. Int J Gynaecol Obstet. 2004; 84(2):120-6.

11. Balat O, Aksoy F, Kutlar I, Ugur MG, Iyikosker H, Balat A, et al. Increased plasma levels of urotensin-II in preeclampsia-eclampsia: a new mediator in pathogenesis?. Eur J Obstet Gynecol Reprod Biol. 2005; 120(1):33-8.

12. López-Jaramillo P, Terán E, de Félix M, Villacres M, Barba L, Moya W Papel del óxido nítrico durante el embarazo normal y la preeclampsia. Rev Colomb Cardiol. 1998; 6:169-76.

13. Chervenak F, Kurjak A. The clinical care of the fetus as a patient. In: Kurjak A, Chervenak F, editors. The fetus as a patient. London: The Parthenon Publishing Group, 1994

14. Merivel P. Physiopatology of pre-eclampsia and intrauterine growth restriction. In: Kurjak A, Chervenak F, editors. The fetus as a patient. London: The Parthenon Publishing Group, 1994. 347-55.

15. Dash PR, Cartwright JE, Baker PN, Johnstone AP, Whitley GS. Nitric oxide protects human extravillous trophoblast cells from apoptosis $\mathrm{B}$ a cyclic GMP-dependent mechanism and independently of caspase 3 nitrosylation. Exp Cell Res. 2003; 287(2):314-24.

16. Neilson JP, Alfirevic Z. Doppler ultrasound for fetal assessment in high risk pregnancies. Cochrane Database Syst Rev. 2010 Jan; (1): CD000073.

17. Papagergeorghiou AT. Predicting and preventing preeclampsia - where to next?. Ultrasound Obstet Gynecol. 2008;31(4): 370- 6

18. Moldenhauer JS, Stanek J, Warshak C, Khoury J, Sibai B. The frequency and severity of placental findings in women with preeclampsia are gestational age dependent. Am J Obstet Gynecol. 2003; 189(4):1173-7

19. Ness RB, Roberts JM. Heterogeneous causes constituting the single syndrome of preeclampsia: a hypotesis and its implications. Am J Obstet Gynecol. $1996 ; 175: 1365-70$

20. Aardema MW, Saro MC, Lander M, De Wolf BT, Oosterhof H, Aarnoudse JG. Second trimester Doppler ultrasound screening of the uterine arteries differentiates between subsequent normal and poor outcomes of hypertensive pregnancy: two different pathophysiological entities?. Clin Sci. (London) 2004; 106: 377-82.

21. Bricker L, Neilson JP, Dowswell T.. Routine ultrasound in late pregnancy (after 24 weeks' gestation). Cochrane Database Syst Rev. 2008 Oct 8;(4):CD001451

22. Vatten LJ, Skjaerven R. Is pre-eclampsia more than one disease? BJOG. 2004; 111(4):298-302

23. von Dadelszen P, Magee LA, Roberts JM. Subclassification of preeclampsia. Hypertens Pregnancy. 2003; 22(2):143-8. 